\title{
Structured Mixed Phase is Favored in Neutron Stars
}

\author{
Michael B. Christiansen \\ Institute of Physics and Astronomy, University of Aarhus, DK-8000 Arhus C, Denmark \\ Norman K. Glendenning \\ Nuclear Science Division and Institute for Nuclear $\&$ Particle Astrophysics, Lawrence Berkeley National Laboratory, MS \\ 70A-319, Berkeley, California 94720
}

(December 10, 2018)

We give a general thermodynamical argument showing that in neutron stars, the Coulomb structured mixed phase is always favored for any first order phase transition involving systems in equilibrium with baryon number and electric charge as the two independent components. This finding is likely to have important consequences for many neutron star properties, e.g., glitch phenomena, transport and superfluid properties, r-mode instabilities, and the braking index.

26.60.+c, 64.70.-p, 97.60.Jd, 68.10.Cr

A number of phase transitions may take place in neutron stars. Described in most detail is the first order nuclear liquid-gas transition in the inner crust of a neutron star, where nuclei with different shapes are in equilibrium with a neutron gas [1.22. Above the nuclear saturation density, phase transitions involving, for instance, quark matter, pion, and kaon condensation have been suggested and described, see e.g., Ref. [3] for an overview.

Before 1992, local charge neutrality was imposed on all the possible phase transitions above saturation density, leaving the systems with only one independent component, namely the baryon number. This treatment made any first order transition resemble, e.g., the water-ice transition. However, in 1992 it was pointed out by Glendenning [4] that because neutron star matter has two independent components, namely the baryon number and the electric charge, charge neutrality must be applied as a global and not a local condition. Gibbs general conditions for thermodynamic equilibrium for both chemical potentials (usually taken to be the neutron and electron chemical potentials) cannot be satisfied otherwise. As a consequence of this, the charge does not vanish identically; rather, there results a nonvanishing charge density of opposite sign for each bulk phase $[5]$.

A first order transition with multiple independent components behaves qualitatively differently from a single component one as seen in Fig. 1. For a one-component system, the transition takes place at a constant pressure where the two phases, having different densities, for all proportions are in equilibrium. The equilibrium pressure is found through a Maxwell construction. Systems with two components have an additional degree of freedom, which means the proportions of the two phases in equi- librium vary as a function of the pressure. The phases still have different densities, but the densities are now also functions of the pressure [4, 6, 67. Mixtures of two miscible liquids in equilibrium with their vapor phase are well described examples from physical chemistry [6, 8]. In a neutron star this means that a two-component phase transition may have a considerable radial extent in contrast to a one-component transition, which takes place at a single radial point in the star, the point where the pressure of the two phases are equal. This region of two coexisting phases in equilibrium is usually referred to as the mixed phase.

In 跑 surface and Coulomb effects were neglected in the description of the mixed phase, although it was pointed out that their inclusion would result in a Coulomb lattice, analogous to the lattice resulting from the nuclear liquidgas transition in the inner crust of a neutron star. Such a structured mixed phase above the saturation density was first studied in 9 for the deconfinement transition. It was there concluded that the mixed phase may not be energetically favored, compared to the locally charge neutral, one-component system, if the surface and Coulomb energies are 'sufficiently large'.

We show in the following that for any first order phase transition of a two-component system of nuclear matter in full thermodynamic equilibrium, the energy (including Coulomb and surface energies) of this system is always smaller than the energy of the same system where one component is frozen out [10]. For a neutron star, the important implication is that for matter in equilibrium there will always be a structured mixed phase if any first order phase transition takes place in its dense interior.

Gibbs conditions for thermodynamical equilibrium between two phases (I and II) with $n$ independent components separated by an arbitrary boundary are [11,12

$$
\begin{aligned}
T^{I} & =T^{I I} \\
\mu_{i}^{I} & =\mu_{i}^{I I} \quad i=1,2, \ldots, n \\
P^{I}\left(\mu_{i}^{I}, T^{I}\right) & =P^{I I}\left(\mu_{i}^{I I}, T^{I I}\right)+\sigma\left(\frac{1}{R_{1}}+\frac{1}{R_{2}}\right)+\ldots,
\end{aligned}
$$

where $T$ is the temperature, $\mu_{i}$ is the chemical potential of the $i$ 'th independent component, $P$ is the pressure, $\sigma$ is the surface tension, and $R_{1}$ and $R_{2}$ are the principal curvature radii (higher order corrections like curvature 
effects have not been explicitly included in the pressure equilibrium condition). These conditions describe thermal, chemical, and mechanical equilibrium, respectively. In addition the energy (as well as the free energies) is at a minimum for a system in equilibrium. All these conditions are direct consequences of the second law of thermodynamics. In the case of a neutron star, Gibbs conditions have to be solved consistently with the condition of charge neutrality, which as emphasized, cannot be imposed as a condition of local vanishing charge density else there are too many unknowns for the number of equations.

Cold nuclear matter in a single phase in equilibrium is of course electrically neutral. $\beta$-equilibrium ensures that the sum of the proton and electron chemical potentials equals the chemical potential of the neutron, $\mu_{n}=\mu_{p}+\mu_{e}$ (the neutrinos escape the system freely). This local charge neutrality requires the electron and proton densities to be equal in bulk. Earlier, local charge neutrality was likewise imposed on both phases of the system during a phase transition. This assumption has the effect of freezing out the electric charge component and leaving the baryon number as the single independent component. The Maxwell construction ensures pressure equilibrium and chemical equilibrium between the baryons across the phase boundary for such a phase transition. However, the electron chemical potential is generally discontinuous across the phase boundary.

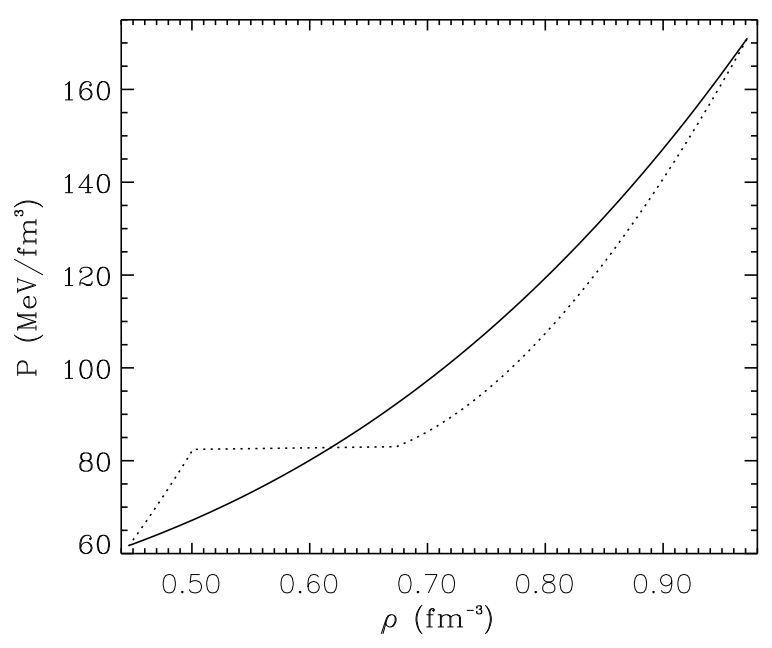

FIG. 1. The pressure as a function of the baryon density in the mixed phase region of a two-component system. The solid curve corresponds to the two-component system, whereas the dotted curve corresponds to the same system with the electric charge component frozen out. The actual numbers are for a system where the first order phase transition is to a kaon condensate [13].

In the current picture of the bulk phases, the local charge neutrality of both phases is relaxed to a global charge neutrality condition, which allows the system to freely explore the additional degree of freedom in a system with two independent components. Compliance with Gibbs phase equilibrium conditions for both chemical potentials naturally result in a nonvanishing charge density for each phase. The normal nuclear matter phase will be positively charged, and thus more isospin symmetric; whereas the high density phase (e.g., quark matter) will be negatively charged, and partly replace electrons (and muons) as global neutralizing agents. The bulk energy of the one-component system lies above the bulk energy of the mixed phase system if Coulomb and surface energies are neglected, since the charge neutrality constraint is relaxed in the latter approach [4].

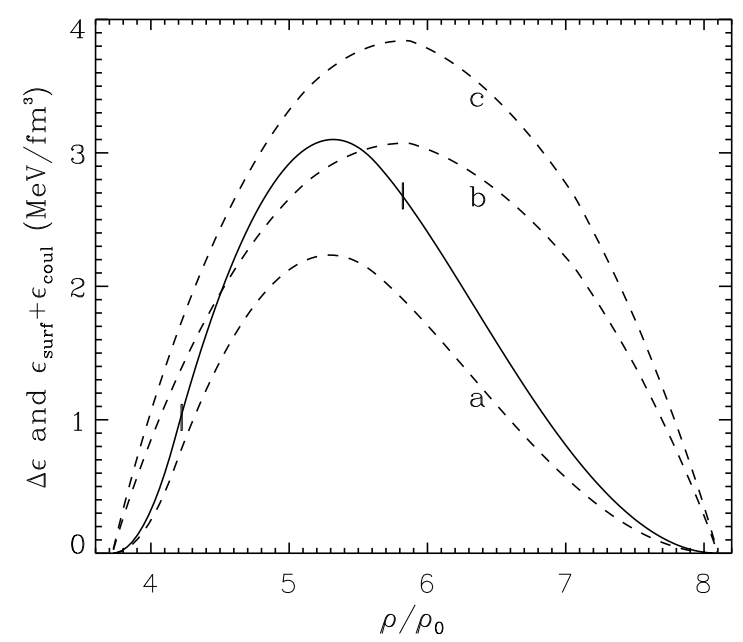

FIG. 2. The solid curve is a schematic plot of the difference in energy density between a Maxwell system and the corresponding bulk mixed phase system as a function of the baryon density in units of the saturation density. The two marks represent the densities of the low and high density phase at the constant transition pressure of the Maxwell system, between which the average baryon density varies linearly according to $\rho=\chi \rho^{I}+(1-\chi) \rho^{I I}$, where $\chi$ is the volume fraction of phase $I$ [15]. The curves $a, b$, and $c$ are schematic plots of the sum of Coulomb and surface energies in the mixed phase region corresponding to the three possible scenarios described in the text.

In the current picture of the structured mixed phase as presented in Ref. [9], the mixed phase is energetically favored only if the sum of Coulomb and surface energies in the mixed phase is smaller than the gain in bulk energy between the two systems. A schematic plot of this comparison is shown in Fig. 2 (see also the figure in [9] or Fig. 30 in 3). There are three possible scenarios, corresponding to the three curves of Coulomb and surface energies: $a$, the structured mixed phase is favored in the whole bulk mixed phase region, $b$, it is favored in some regions of the bulk mixed phase, $c$, it is not favored anywhere. The properties of the equations of state including 
surface and screening effects for the two phases determine which scenario is valid for the phase transition in question. Screening effects reduce the Coulomb energy and will play an increasingly important role with increasing size of the charged structures. Screening lengths for different components have been estimated, the ranges are from about $5 \mathrm{fm}$ to $13 \mathrm{fm}$. Structures that are smaller than the screening lengths will have almost uniform particle densities within each phase and a nonvanishing local charge density. On the other hand, the larger the structures are compared to the screening lengths, the more the system will resemble two electrically neutral bulk phases [9]. 3].

The new picture we present is based on the thermodynamical equilibrium conditions described in Eq. (11) and below. These conditions are direct consequences of the second law of thermodynamics and therefore have general validity. The standard Maxwell construction can only ensure mechanical equilibrium and one chemical potential to be in equilibrium across a phase boundary. The other chemical potential will generally be discontinuous across the boundary. Thus, if one component is frozen out by imposing constraints on a system with two independent components, this system cannot be in full thermodynamical equilibrium. Therefore the (free) energy of such a system is not at a minimum, and there must be another system in thermodynamical equilibrium in which the energy is a global minimum. This statement is valid over the whole range of the mixed phase region and not only at the pressure $P_{t}$, where the actual discontinuity in one chemical potential is. To see this consider the system (I) in the neutron star with the global energy minimum at $P_{t}$ and assume that the single phase (II) with the imposed local charge neutrality is preferred at an infinitesimal smaller (or larger) pressure. There will be a phase boundary between these phases, but they will have different chemical potentials and therefore will only be in mechanical equilibrium. Thus there must be a system different from (II) at this pressure which is in thermodynamical equilibrium with (I) and being at the global energy minimum. This argument can be repeated until the whole mixed phase region has been spanned. Notice, that the above argument is valid irrespective of whether the central pressure of the neutron star is larger than $P_{t}$ of the Maxwell system or not. Therefore, a two-component system with one component frozen out cannot be at a global energy minimum since it is not in full thermodynamical equilibrium. The system with the global energy minimum, in the pressure region where the equations of state indicate a two-phase equilibrium is possible, must therefore be the mixed phase with the particular Coulomb lattice structure which has the lowest energy, since this is the only system which is in full compliance with Gibbs equilibrium conditions.

Screening and surface effects do not change the above conclusion. Phase boundaries are inevitable in two-phase systems in equilibrium, and likewise are screening effects an integral part of all charged systems in equilibrium, whether the systems consist of a single phase or two (or more) phases separated by a phase boundary. In the latter case screening and surface effects across the phase boundary become inseparable. Charged systems larger than the typical screening length can reduce their Coulomb energy by reducing the local charge density. But this does not imply that these large systems will generally resemble that of a one-component system with the electric charge frozen out. As long as the charge density does not vanish identically everywhere in the systems, the Wigner-Seitz cells will survive 14. On this basis the scenarios $b$ and $c$ in Fig. 2 can be excluded for any first order phase transition of such two-component systems in full thermodynamical equilibrium.

For a given phase transition a figure like Fig. 2 is very useful though. If the model describing the structured mixed phase has an energy corresponding to scenario $b$ or $c$, we can immediately conclude that the model does not give a correct description, since we already know the Maxwell-like, one-component approximation represents an excited state. This is a powerful test of whether the approximations in the treatment of Coulomb and surface energies are good enough. An important implication is that a rough upper limit on the surface tension in a given model can be estimated from this comparison. However, one should be careful not to use (reasonable) guesses for the value of the surface tension to draw conclusions about the physical possibilities of the system as it was done in [9]. We note that in the description of the first order nuclear liquid-gas transition, the findings of Ref. [2] are that the structured mixed phase is indeed favored except close to the upper end of the mixed phase region. Thus, their model gives at least a physically sound description of the structures in most of the mixed phase region except close to its upper end where the details of the model, here becoming increasingly important, can be improved upon.

There are situations where scenario $b$, if it had been favored, could have led to buoyancy instabilities analogous to a situation where mercury is placed on top of liquid water. Assume for example that the structured mixed phase for the system shown in Fig. 11 is favored only if the pressure is less than $65 \mathrm{MeV} / \mathrm{fm}^{3}$ corresponding to baryon densities below $0.48 \mathrm{fm}^{-3}$. Since hydrostatic equilibrium requires that the pressure increases monotonically from the surface to the center of the neutron star, there will be a region in the star where a denser structured mixed phase is located further from the center than a less dense Maxwell-like phase. This is a gravitationally unstable situation commonly seen in some stars. A similar situation arises within the structured mixed phase, but here the two phases in each Wigner-Seitz cell have a non-vanishing charge density, and the Coulomb force which is much stronger than gravity stabilizes the cell and secure that it remains intact. 
In specific studies of possible phase transitions, reasonable models for the equations of state of the phases are crucial. For some choices of models (or parameter values within a model) it may happen that it is not possible to assure full compliance with Gibbs equilibrium conditions, even if a Maxwell construction, indicating the transition is first order, can be found. Examples relating to kaon condensation can be found in 16. Since the Maxwell construction represents an excited state in a two-component first order phase transition, the model must be unphysical since by construction it cannot describe the system in full thermodynamical equilibrium. We note that the authors of Ref. 16 are aware of this.

There is a single case where the transition can be first order without there being mixed phase. If it happens that also the electron chemical potential is continuous at the constant pressure transition of the Maxwell-like system, then there will be no mixed phase (or more precise, the radial extent of the mixed phase region has shrunk to nothing). But this is by no means related to screening effects or energy considerations between the Maxwell-like system and the mixed phase. It would be a completely coincidental property of the two phases and the equations of state describing them, and therefore it has vanishing probability.

We have, based on Gibbs equilibrium conditions, presented a new picture showing that in neutron stars, the structured mixed phase will always be energetically favored for arbitrary first order phase transitions involving systems in full thermodynamical equilibrium with baryon number and electric charge as the two independent components. Therefore, models that impose local charge neutrality on such two-component systems and thereby make the phase transition resemble that of one-component systems cannot be physically sound. Additionally, we have shown a way of checking whether the applied models are physically reasonable.

This conclusion is likely to have important consequences for a broad range of neutron star properties, e.g., glitch phenomena, transport and superfluid properties, r-mode instabilities, and the braking index, see [3] and references therein. Details concerning these phenomena depend of course on the specific properties of any first order phase transition taking place in the dense interior of neutron stars, especially the radial extent of the mixed phase region, the density at which it starts forming, and whether unsurmountable energy barriers prevent the neutron star matter from actually achieving its equilibrium configuration.

M.B.C. thanks Jes Madsen for useful discussions. M.B.C. was supported in part by the Carlsberg Foundation and Direktør Ib Henriksens Fond. N. K. G. was supported by the Director, Office of Science, Office of High Energy and Nuclear Physics, Division of Nuclear Physics, and by the Office of Basic Energy Sciences, Division of Nuclear Sciences, of the U.S. Department of
Energy under Contract No. DE-AC03-76SF00098.

[1] C. J. Pethick and D. G. Ravenhall, Ann. Rev. Nucl. Part. Sci. 45, 429 (1995), and references therein.

[2] C. P. Lorenz, D. G. Ravenhall, and C. J. Pethick, Phys. Rev. Lett. 70, 379 (1993).

[3] H. Heiselberg and M. Hjorth-Jensen, Phys. Rep. 328, 237 (2000).

[4] N. K. Glendenning, Phys. Rev. D 46, 1274 (1992), see also N. K. Glendenning, Nucl. Phys. B (Proc. Suppl.) 24B, 110 (1991).

[5] N. K. Glendenning, COMPACT STARS, Nuclear Physics, Particle Physics, and General Relativity (SpringerVerlag, New York, 1997) p. 308.

[6] H. B. Callen, Thermodynamics and an introduction to thermostatistics, 2nd ed. (Wiley, New York, 1985).

[7] This continuous variation in composition with pressure has misled some people to describe such a two-component phase transition as an overall second order transition with local first order characteristics [3], but it is simply the characteristics of a first order phase transition in a multi-component system.

[8] J. O. Hirschfelder, C. F. Curtiss, and R. B. Bird, Molecular Theory of Gases and Liquids (Wiley, New York, 1954).

[9] H. Heiselberg, C. J. Pethick, and E. F. Staubo, Phys. Rev. Lett. 70, 1355 (1993).

[10] This statement is stronger than the general physical principle stating that opening a degree of freedom to a system has the effect of lowering the energy of the system, or leaving it unchanged, not raising it; because the system may already be in its optimum configuration with respect to this degree of freedom, which leaves the energy of the system unaltered.

[11] J. W. Gibbs, The Collected Works, Vol. I, (Yale University Press, New Haven, 1957).

[12] E. A. Guggenheim, Thermodynamics an Advanced Treatment for Chemists and Physicists, 2nd ed. (NorthHolland, Amsterdam, 1950).

[13] N. K. Glendenning and J. Schaffner-Bielich, Phys. Rev. Lett. 81, 4564 (1998).

[14] If the charge density vanishes identically in a WignerSeitz cell, the two phases will separate due to gravity. But because of the infinitesimal difference of the gravitational field across the dimension of the cell, this will only happen if the local charge density is vanishing.

[15] In contrast to the figure in Ref. [9] we also include the energy difference between the two systems in the region, where the average baryon density of the Maxwell system varies linearly. The energy density of the mixed phase is not constant in this region, and therefore the curve between the two marks is not a straight line. Notice also that the surface energy corresponding to the phase boundary in the Maxwell system has been ignored previously. We do not include it either, but note that this energy, if included in the energy difference curve, will add an additional contribution in the region between the marks.

[16] J. A. Pons, S. Reddy, P. J. Ellis, M. Prakash, and J. M. Lattimer, nucl-th/0003008. 